\title{
Um ensaio sobre a cegueira: saúde mental na atenção básica e as disputas diante da pandemia da covid-ı
}

\section{An essay on blindness: mental health in primary care and disputes in the face of the pandemic of covid-19}

\author{
Mússio Pirajá Mattos \\ (D) https://orcid.org/0000-0002-8792-5860 \\ E-mail: mussio.mattosळufob.edu.br \\ Beatriz Medrado Pereira ${ }^{a}$ \\ (D) https://orcid.org/0000-0003-1148-4648 \\ E-mail: beatriz_medradoळhotmail.com

\section{Daiene Rosa Gomes ${ }^{a}$} \\ (D) https://orcid.org/0000-0002-1831-1259 \\ E-mail: daiene.gomesळufob.edu.br
}

anúcleo de Pesquisa em Saúde Coletiva. Centro das Ciências Biológicas e da Saúde. Universidade Federal do Oeste da Bahia. Barreiras, BA, Brasil.

\section{Correspondência}

Daiene Rosa Gomes

Universidade Federal do Oeste da Bahia. Rua Professor José Seabra de Lemos, 316, pavilhão de aula 2, gabinete 38. Barreiras, BA, Brasil. CEP 47808-02।.

\section{Resumo}

A atenção básica à saúde (ABS) é um importante recurso para o enfrentamento de problemas relacionados ao sofrimento psíquico. A partir disso, o presente ensaio visa discutir a saúde mental (SM) na ABS diante da pandemia da covid-19. A pandemia ilumina problemas antigos em que é observado a lógica assistencialista e medicalizada do cuidado em SM. Além disso, persistem práticas de subaproveitamento e não reconhecimento das potencialidades da ABS como produtora do cuidado num espinhoso cenário político-econômico de disputa de poder e que incentiva a privatização dos serviços públicos. Esse jogo de forças tem influência na produção do cuidado, sobretudo em contextos caóticos, de injustiças e desigualdades sociais, terrenos férteis para a disseminação da covid-19. Assim, torna-se importante um olhar mais atento sobre os efeitos da SM na ABS, visto que gestores, profissionais e usuários podem mergulhar na "cegueira branca". Portanto, é fundamental reconhecer a legitimidade dos saberes, valores e desejos que movimentam os trabalhadores e usuários dos serviços públicos; ou seja, compreender a SM como um campo relacional e dinâmico. Também é importante depositar um sopro de esperança ao gerar reflexões que acendam as luzes para que esse cuidado se aproxime do cuidado integral.

Palavras-chave: Atenção básica à saúde; Políticas de saúde; Cuidado; Saúde mental; Covid-19. 


\section{Introdução}

Primary health care (PHC) is an important resource for facing issues associated with psychological distress. Thus, this essay aims to discuss mental health $(\mathrm{MH})$ in PHC in the face of the covid-19 pandemic. Besides bringing to light old problems related to the assistentialist and medicalized logic of the $\mathrm{MH}$ care, the pandemic upholds practices that underutilize and disregard PHC potentialities as a care provider in a political-economic scenario of power struggle, which encourages the privatization of public services. Such power play influences care provision, especially in chaotic scenarios of injustices and inequalities - fertile ground for covid-19 spread. This context evinces the need for taking a closer look at the effects of MH on PHC, since managers, professionals, and users can be plunged into the so-called "white blindness". Thus, recognizing the legitimacy of knowledge, values, and desires that move different types of workers and users is crucial; that is, understanding $\mathrm{MH}$ as a relational field in constant movement. Likewise, bringing a glimmer of hope by promoting reflections that turn on the lights so that this care approaches integral care is also important.

Keywords: Primary health care; Health policy; Care; Mental health; Covid-19.
No Brasil, tem-se observado um aumento considerável da abrangência da Estratégia Saúde da Família (ESF) na atenção básica à saúde (ABS), fruto dos processos de Reforma Sanitária e Reforma Psiquiátrica Brasileira (RPB) (Dalla Vecchia; Martins, 2009). Trata-se de um aspecto bastante relevante, uma vez que a ABS é considerada uma ferramenta estruturante dos sistemas de saúde e um eixo fundamental para a construção de uma atenção continuada e integral em saúde. Assim, o papel dos profissionais da ABS é fundamental para o alcance dos objetivos de garantia de direitos humanos e qualificação assistencial à população (Cecilio; Reis, 2018; WHO, 2008).

O relacionamento contínuo entre profissional de saúde e paciente é um elemento central para se efetivar a ABS. No campo da saúde mental (SM), como parte integrada desses serviços, os transtornos mentais são mais facilmente identificados e tratados, atingindo, assim, bons resultados terapêuticos (WHO, 2008). Nesse sentido, o presente ensaio adota a terminologia de ABS fundamentada nos princípios do Sistema Único de Saúde (SUS), que busca garantir a universalidade, integralidade e equidade dos cuidados primários (Cecilio; Reis, 2018).

A aplicação do princípio da integralidade do cuidado em SM na ABS pode ser viabilizada segundo um modelo de rede de cuidados, do qual os Centros de Atenção Psicossocial (CAPS) não são os únicos dispositivos (MS, 2003). As equipes de ABS possuem proximidade com as famílias e as comunidades, além de serem um importante recurso para o enfrentamento de problemas relacionados ao uso abusivo de drogas e outras formas de sofrimento psíquico (MS, 2005). Entretanto, no campo da SM, há dificuldades na produção do cuidado na ABS pela persistência da dicotomia entre problemas da mente e do corpo, incentivada pela formação tecnicista e por complexidades em que a psiquiatria clássica, por si só, não pode oferecer solução (Bezerra et al., 2014; Dalla Vecchia; Martins, 2009).

Nessa direção, persistem práticas de subaproveitamento e de não reconhecimento das potencialidades da ABS como produtora do cuidado em SM num espinhoso cenário político-econômico de disputa de poder 
(Bravo; Pelaez; Menezes, 2019). Esse jogo de forças influencia na produção do cuidado, sobretudo em contextos caóticos como o da pandemia da covid-19, em que o ato de cuidar traz implicações à SM de usuários, bem como dos profissionais de saúde que atuam na linha de frente dos serviços (Ornell et al., 2020).

Além disso, o Governo Federal negou os impactos da pandemia na população e foi ausente em seu enfrentamento, de modo que foi conferida autonomia aos estados para que pudessem adotar as devidas medidas protetivas. A negligência do Governo Federal perante o combate ao coronavírus conferiu espaço para a iniciativa privada se instalar na gestão da epidemia (Sodré, 2020).

Assim, há uma perpetuação da cultura política e clientelista que acompanha o processo histórico do governo brasileiro e sua atuação no controle das massas, em que os direitos e o bem-estar da população são abandonados (Schünke; Giongo, 2018), de tal forma que os gestores, os profissionais e os usuários dos serviços públicos de saúde podem mergulhar na "cegueira branca" (Saramago, 1995), que é talvez o pior tipo de cegueira que existe, uma vez que, nela imersos, não se enxerga a própria essência. Entende-se pela obra de Saramago (1995) que tal cegueira é a da alienação e do egoísmo, que nos impedem de enxergar os problemas que nos afligem enquanto sociedade. Por esse motivo, consideramos que o país esteja imerso numa pandemia de "cegueira branca" no que se refere aos assuntos relacionados às garantias dos direitos da população.

Outro importante ponto de discussão implica em reconhecer que pessoas diagnosticadas com covid-19 também estão susceptíveis a algum tipo de sofrimento mental. Nesse contexto, é importante estar mais atento aos efeitos da SM na ABS, uma vez que os transtornos mentais são uma parcela significativa da demanda da ABS, e, particularmente durante uma pandemia, o sofrimento psicológico pode ser amplificado. Em um estudo transversal realizado com 45.161 brasileiros durante a pandemia da covid-19, verificou-se que $40,4 \%$ se sentiram tristes ou deprimidos; $52,6 \%$ frequentemente ansiosos ou nervosos; $48 \%$ relataram problemas de sono preexistente agravado (Barros et al., 2020). Dessa forma, as elevadas prevalências apresentadas indicam a crescente necessidade de garantir serviços de atenção à SM no contexto pandêmico.

A fim de contribuir para a solução desse desafio, este ensaio visa discutir sobre a SM na ABS no contexto da pandemia da covid-19. Para isso, buscou-se evidenciar a ABS como a força motriz na produção do cuidado em SM; as relações de poder, bem como os espinhosos atravessamentos políticos e econômicos que permeiam as decisões em nosso país; e a tragédia sanitária e suas implicações na perturbação psicossocial, exigindo novas formas de atender e cuidar da SM da população. O texto está organizado em duas seções em que serão apresentados os desafios do cuidado em SM na ABS e a negligência perante as injustiças e as desigualdades sociais, terrenos férteis para disseminação da covid-19.

\section{A cegueira por detrás da espinhosa arena de disputas: interlocução entre a saúde mental e a atenção básica à saúde no contexto da pandemia da covid=19}

As forças que operam no cotidiano constituem campos de disputa relacionados com a micropolítica, relações de poder e relações intersubjetivas. São nos encontros entre gestores, trabalhadores e usuários que se instauram os campos de força que são produzidos por contínuos tensionamentos (Merhy et al., 2019). Desse modo, onde há uma força atuando em um sentido, sempre haverá outra operando no sentido contrário, isto é, resistindo, o que produz tensão política, econômica e social. Discutiremos esse contexto por meio das seguintes questões: "Afinal, a quem cabe superar o poder de decisão do seu próprio destino?" "Como superar o sofrimento de enxergar enquanto insistem em permanecer cegos nessa espinhosa arena de poder?" Trata-se de questionamentos importantes para o entendimento do contexto da pandemia do coronavírus e da decorrente amplificação das desigualdades sociais e das disputas políticas dessa crise sanitária.

A pandemia iluminou problemas antigos de nossa sociedade. Nessa direção, em um trabalho de revisão, Gryschek e Pinto (2015) apontam que as comunidades 
em que a ESF se insere são áreas pobres e que contam com poucos recursos comunitários e sociais, sendo comum o consumo de drogas, a violência e o desemprego, associados aos problemas de SM. Além disso, as equipes da ESF não compreendem suas práticas de atendimento à população como cuidado em SM e, por vezes, se consideram incapacitadas para aprendê-la adequadamente devido à persistência do modelo biomédico e positivista em sua formação e atuação profissional. Entre as circunstâncias que consideram mais difíceis no atendimento estão os casos de tentativa de suicídio e episódios psicóticos. Segundo eles, o cuidado nesses casos é focado na medicação e no encaminhamento para avaliação especializada. Dessa forma, instrumentos de cuidado, como o acolhimento e a escuta, são pouco explorados em sua prática cotidiana.

Outro aspecto relevante é a lógica assistencialista e medicalizada da SM na ABS. A medicamentalização se refere ao controle médico sobre a vida das pessoas e representa um risco do aumento do poder da psiquiatria, da psicologização dos problemas sociais e da ampliação da ambulatorização (Bezerra et al., 2014), que são formas de cuidar privatizantes e que contradizem as conquistas da RPB. É possível enxergar que, apesar dos avanços de novas concepções epistêmicas acerca dos conceitos de saúde-doença, SM e cuidado-cura, o modelo psiquiátrico tradicional ainda não foi superado pelo modelo psicossocial (Sampaio; Bispo Júnior, 2021). É importante destacar que não se trata de desqualificar a importância do uso do medicamento, mas em criticar os movimentos de patologização da vida e de continuidade da lógica biologicista.

Um dos grandes desafios da Política Nacional de Saúde Mental (PNSM) brasileira é a formação de profissionais para o trabalho interdisciplinar e intersetorial capazes de produzir a superação do paradigma da tutela, do estigma e do preconceito (MS, 2005). Sampaio e Bispo Júnior (2020), ao revisitar o percurso histórico da institucionalização da SM no Brasil, compreendem que a luta da RPB é também a luta pelo direito ao exercício da cidadania, por uma sociedade mais igualitária, pela horizontalização do poder em arenas de conflitos e pela ocupação de espaços para dar voz aos que vivenciam o sofrimento mental.
Essa luta é relevante porque o poder que repercute no campo da SM na ABS atinge a configuração micropolítica do "maior-saber-maior-poder", pelo qual o conhecimento se fortalece para a detenção de poder em uma arena de conflitos e interesses políticos, concedendo aos mais fortes a capacidade de reprimir e exigir obediência daqueles que sabem menos (Silva et al., 2019). Esse poder no campo da SM, no qual se vivencia a ideia do poder disciplinar para aqueles que possuem comportamentos ditos "diferentes", contribui para o processo histórico de exclusão. As relações de poder estão diretamente relacionadas a aspectos políticos, sobretudo as discussões que envolvem a organização de novas práticas de produção do cuidado em SM. Desta forma, a manutenção da institucionalização e das práticas disciplinares tornam-se mais cômodas e asseguram o exercício do poder dos políticos, gestores e demais profissionais (Arejano; Padilha; Albuquerque, 2003).

0 trabalho e a gestão dos serviços públicos de saúde são guiados por motivações políticopartidárias, engrenagens para a nomeação de cargos públicos, com fins eleitoreiros e de manutenção do poder (Schünke; Giongo, 2018). Nos últimos quatro anos, o Brasil sofreu uma restauração ultraconservadora de projetos políticos neoliberais, autoritários e pró-capital, que centram esforços na garantia dos interesses das classes dominantes (Bravo; Pelaez; Menezes, 2019). 0 foco da vida pública deixa de ser a defesa dos direitos universais da população e passa a ceder espaço ao desmonte da saúde pública e da proteção social, algo grave num cenário de congelamento por vinte anos de recursos financeiros e cortes orçamentários para as políticas sociais, além das propostas de retrocessos na política de SM, que ameaçam as conquistas da RPB no país, mudanças na Política Nacional de Atenção Básica e avanço do setor privado da gestão dos serviços (Daumas et al., 2020; Sodré, 2020).

Nesse ínterim, em 2019, o Ministério da Saúde direcionou suas ações para políticas de privatização ou cortes orçamentários nos programas existentes, de modo que se passou a questionar a gratuidade e a universalidade do SUS, o que corrobora para a ameaça ao sistema de saúde pública brasileiro. Desta forma, Sodré (2020) discute algumas ações que foram materializadas, como o esvaziamento do 
Núcleo Ampliado de Saúde da Família; retomada do Programa Mais Médicos, atendendo a demanda do Revalida por meio da expansão de parcerias para receber médicos recém-formados pelas faculdades privadas, ao mesmo tempo em que ampliou o embargo de médicos estrangeiros; e a criação de um serviço autônomo para gestão da ABS, a Agência para Desenvolvimento da Atenção Primária à Saúde, que visa retirar do SUS a administração direta dela.

Portanto, as reduções e cortes no financiamento em saúde prejudica a capacidade de uma resposta eficiente e diligente à pandemia da covid-19. Essa tragédia sanitária, assim como as medidas de isolamento e distanciamento social, jogou luz sobre os olhares das desigualdades pré-existentes. O negacionismo cresceu e, ao negar a gravidade da pandemia e, consequentemente, os cuidados quanto a ela, a extrema-direita minimiza a importância crucial das políticas públicas, eximindo o Estado de investir na saúde pública. A consequência mais cruel dessa cegueira é o fortalecimento de uma política de morte sobre os grupos mais vulneráveis: negros, pobres, idosos, povos indígenas e mulheres (Morel, 2021).

Quanto a SM, uma amostra realizada no Brasil, composta por indivíduos entre 18 e 75 anos, todos alfabetizados, indicou que, durante a pandemia da covid-19, ter a renda diminuída, fazer parte do grupo de risco e estar mais exposto a informações sobre mortos e infectados são fatores que podem provocar maior prejuízo psicológico (Duarte et al., 2020). Portanto, o contexto da pandemia é um momento para estimular esforços visando minimizar os aspectos mais negativos da SM na população. Por esse motivo, acreditamos que aproximar a SM da ABS tem o potencial para iluminar o olhar e fomentar ações e reflexões para novos modos de cuidar da saúde pública, mesmo que seja nos "olhos do furacão".

\section{Produzir cuidado no olho do furacão:} - encontro entre saúde mental, atenção básica à saúde e a pandemia da covid= 19

A discussão anterior permite perceber que o poder não é algo que se concentra ou se possui, é uma estratégia de localização da ordem relacional entre homens ou grupos de homens; é algo que se exerce e se apoia nos despossuídos, ao invés de desapossar (Foucault, 1995). Nesse encontro, cabem novas interrogações: "Vivemos em uma sociedade de cegos?" "Qual o sentimento que se deve ter em ver a degradação provocada por uma pandemia de cegueira branca?" "Afinal, ao entrar no olho do furacão, é possível enxergar uma tempestade que deixa a população do país à deriva frente a um impacto psicossocial em diferentes níveis e gravidades?"; além disso: "Pessoas com transtornos mentais anteriores à pandemia estão entre os grupos vulneráveis para o agravamento dos sintomas?" (Fiocruz, 2020).

Esse contexto também requer maior atenção ao trabalhador da saúde, pois ele está mais sujeito a ter sua SM afetada pelas situações cotidianas de trabalho. É recorrente o aumento dos sintomas de ansiedade, depressão, perda da qualidade de sono, aumento do uso de drogas lícitas e ilícitas, sintomas psicossomáticos e medo de se infectar ou transmitir a infecção aos membros da família. Os familiares dos profissionais de saúde também estão mais propensos a apresentarem sofrimento psíquico durante a pandemia (Fiocruz, 2020). Essa tragédia sanitária tem sido acompanhada pelo medo, estresse e pânico generalizado muito em função da crise político-institucional do país.

Nessa direção, foi elaborado um material que reuniu sugestões de cuidado em SM alinhadas com as recomendações da Organização Mundial da Saúde (OMS) (WHO, 2020), dentre elas: reconhecer as emoções e aceitá-las, mas evitar sofrimentos desnecessários com excesso de informações; não discriminar ou contribuir para que um conhecido ou familiar que apresente sintomas de covid-19 sinta-se culpado; proteger as crianças, sem fomentar o medo ou o pânico; acolher e proteger os idosos; reduzir as responsabilidades e as exigências do cotidiano e passar mais tempo próximo dos entes queridos; manter rotina prazerosa e significativa; se o ambiente doméstico for violento e hostil, denunciar ou pedir ajuda a rede sócio-assistencial; fazer exercícios físicos em ambientes protegidos; e manter a fé e as atividades religiosas/espirituais.

O encontro entre SM e ABS no olho do furacão tenta estabelecer fortalezas para a oferta de cuidados. 
Diante da pandemia do coronavírus, a organização de estratégias psicossociais é emergente e, para garantir o cuidado em SM na comunidade, recomenda-se a execução de ações na ABS como: identificar, via telefone ou visita domiciliar, as famílias que apresentam fatores de risco relacionados a maior adoecimento mental; identificar as famílias em maior vulnerabilidade e promover a articulação intersetorial para viabilizar resposta às demandas; orientar a população com informações que minimizem o adoecimento mental durante o confinamento; e apoio para minimizar as barreiras para vivência do luto daqueles que perderam os entes queridos (Nabuco; Oliveira; Afonso et al., 2020). Assim, são apresentadas propostas para atuação das equipes da ABS no enfrentamento ao adoecimento mental relacionado à pandemia.

No centro dessa discussão, indagamos: "Como resistir e produzir cuidado frente a uma política de desmonte e privatização?” A gestão privada, como se sabe, transforma o que é direito em um privilégio para poucos, isto é, para aqueles que podem pagar. Ainda assim, para retomar a defesa do SUS, é necessário resgatar a constatação de que os profissionais de saúde devem ser capacitados para detectar problemas de SM no território e propor formas de intervenção adequadas, de modo a criar cooperação e coordenação entre a ABS e o cuidado especializado. Além disso, a literatura cientifica também apresenta o apoio matricial como uma ferramenta adequada para capacitar a ABS para o desenvolvimento de habilidades fundamentais em SM (Gryschek; Pinto, 2015). Entretanto, trata-se de um arranjo complexo, devido aos constantes desmontes que o sistema de saúde está sofrendo.

Destacamos, então, o desafio do SUS em construir a política e, ao mesmo tempo, os atores dela. Desta forma, práticas efetivas de cogestão, educação permanente e apoio matricial encontram dificuldades em obter subsídios para aprimorar uma política educativa em SM no Brasil. Assim, por se tratar de um fenômeno multifacetado, torna-se importante o reconhecimento de mudança institucional e de transformação à luz da RPB e da PNSM (Mattos et al., 2020). O SUS precisa produzir agentes transformadores e formadores para produzir os atores que vão se engajar em uma nova política. Nessa permanente arena de disputas de sentidos, que é a construção das políticas públicas no país, propostas para a gestão do SUS estão sempre presentes e disputando sentidos (Cecilio; Reis, 2018).

Ao se deparar com essas práticas e olhar em direção aos movimentos da RPB, é impossível não se afetar. É importante problematizar esse cenário para produzir práticas em SM sem desviar os nossos olhares; ou seja, sem considerar as necessidades locais e/ou instituir olhares idealizados na acadêmica que podem dificultar a leitura das especificidades do cotidiano dos serviços e da produção de novas formas de cuidar (Lima, 2011).

Nesse compartilhamento de horizontes, torna-se fundamental revisitar a grandiosidade do cuidado levantado por Ayres (2009, p. 36): “cuidar é querer, é fazer projetos, é moldar a argila. É sustentar no tempo, contra e a partir da resistência da matéria, uma forma simplesmente humana de ser". Assim, é permitido enxergar um processo de encontro de culturas que produz novas formas que se combinam de maneiras diferentes. Essa diversidade possibilita a construção de autonomias possíveis a partir da singularidade dos sujeitos, o que contribui para que a noção de cura seja repensada.

Dessa forma, a lógica do cuidado em SM não segue o caminho de sintomas-diagnóstico-terapêutica-cura, pois o sintoma nunca se elimina, ele circula (Lancetti; Amarante, 2008). Cuidar abrange as relações com os outros, algo que envolve o comprometimento e empatia nos espaços de encontro. Cuidar, nesse sentido, representa estar em um processo relacional de constante invenção, construção, costura, montagem de projetos de vida singulares; é agenciar e articular um espaço intersubjetivo (Lima, 2011).

Acreditamos que tais processos são formas de conseguir enfrentar os modelos hegemônicos que se concentram em fenômenos patológicos e político-institucionais. Desta forma, torna-se importante problematizar para avançar na produção do cuidado, em especial, no campo da RPB, com o olhar por relações horizontalizadas e por meio de diálogos, escuta acolhedora, responsabilização de profissionais e usuários em direção a um comprometimento político com as mudanças sociais e culturais (Ayres, 2009; Lima, 2011). 
A produção do cuidado no olho do furacão exige o encontro de soluções melhores e menos cristalizadas para com os usuários. Assim, para não ser arremessado de um lado para o outro na aceleração da pandemia, é importante evocar a nossa capacidade de autoanálise, de repensar nossas práticas, de nos colocar em dúvida, de experimentar coletivamente. A análise do conjunto de práticas produzidas é necessária para poder estruturar e reinventar novos espaços dentro e fora do circuito que o furacão poderá percorrer (Almeida et al., 2020).

Para que isso aconteça, é necessário que a dúvida seja um elemento possível, que seja acolhida e considerada em sua potencialidade no enfrentamento entre forças que marcam as disputas (Almeida; Merhy et al., 2020; Merhy et al., 2019). Nesse caminho, torna-se fundamental admitir que o trabalho vivo exerce o governo de si, com alto grau de autonomia, apesar dos desígnios da gestão e demanda dos serviços.

Assim, é importante reconhecer a legitimidade dos saberes, valores e desejos que movimentam os diferentes tipos de trabalhadores e usuários; ou seja, compreender a produção do cuidado em SM como uma micropolítica, como um campo relacional dinâmico (Merhy et al., 2019). Isso é imanente às relações humanas, pois elas necessitam um constante fazer e refazer para o controle de corpos. Nesse sentido, observa-se o desafio de produzir cuidado em SM no cenário de sofrimento e contradições. Por outro lado, é importante depositar um sopro de esperança ao gerar reflexões que acendam as luzes para que esse cuidado se aproxime do cuidado integral.

\section{Considerações finais}

Em face do que foi discutido, observa-se como a saúde pública no Brasil tem sido resistência em meio aos ataques à sua existência, dado que a SM e ABS são dois eixos advindos de lutas constantes para se implantar, o que torna ainda mais agravante ao pensar na necessidade de sua interlocução. Parafraseando Saramago (1995), estamos cegos; somos cegos que veem, cegos que, vendo, não veem. Cegos que se mascaram ou são mascarados diante da espinhosa arena de poder. Ou seja, a nossa cegueira não é patologia biológica, mas patologia da alma, pois só vemos aquilo que desejamos e vestimos com a invisibilidade aquilo que não queremos enxergar.

Neste cenário, percebe-se a emergência da produção do cuidado em SM, da integralidade e da articulação da ABS no SUS. Para tanto, deve-se superar os aspectos relacionados aos campos de disputa econômica, em que as forças governamentais estão exclusivamente voltadas, ameaçando o protagonismo da ABS para a produção de cuidado psicossocial por meio dos cortes financeiros à saúde pública. Por isso, é preciso despir nossos olhos e ampliar nosso olhar na tentativa de fortalecer a produção do cuidado em SM na ABS, bem como fortalecer os trabalhadores, usuários e, assim, alcançar uma saúde pública mais participativa e inclusiva.

Portanto, é importante limpar as lentes e retirar as películas da desgovernança do cuidado, impedindo que governos impetuosos alcem poder e nos mascarem, para que, assim, se possa enxergar a importância dos equipamentos de saúde, do nosso SUS e da ABS - ferramenta norteadora para superar a crise de saúde pública ocasionada pela pandemia da covid-19. Somente assim, com olhos ávidos e atentos, poderemos mudar o curso dessa tempestade e alcançar novos caminhos, em que os nossos direitos, saúde, bem-estar e proteção sejam de fato a prioridade de nossos governos.

\section{Referências}

ALMEIDA, S. A.; MERHY, E. E. Micropolítica do trabalho vivo em saúde mental: composição por uma ética antimanicomial em ato. Psicologia Política, São Paulo, v. 20, n. 47, p. 65-75, 2020. Disponível em: <http://pepsic.bvsalud.org/pdf/rpp/ v2on47/v2on47ao6.pdf >. Acesso em: 10 mar. 2021.

AREJANO, C. B; PADILHA, M. I. C. S;

ALBUQUERQUE, G. L. Reforma psiquiátrica: uma analítica das relações de poder nos serviços de atenção à saúde mental. Revista Brasileira de Enfermagem, Brasília, DF, v. 56, n. 5. 2003. Disponível em: <https://www.scielo.br/pdf/reben/ v56n5/a16v56n5.pdf>. Acesso em: 2 fev. 2021.

AYRES, J. R. C. M. Cuidado: trabalho e interação nas práticas de saúde. Rio de Janeiro: Roseni Pinheiro, 2009. (Clássicos para Integralidade em Saúde).

BARROS, M. B. A. et al. Relato de tristeza/ depressão, nervosismo/ansiedade e problemas 
de sono na população adulta brasileira durante a pandemia da covid-19. Epidemiologia e Serviços de Saúde, Brasília, DF, v. 29, n.4, p. e2020427, 2020. Disponível em: <http://scielo.iec.gov.br/pdf/ess/ v29n4/2237-9622-ess-29-04-e2020427.pdf>. Acesso em: 8 mar. 2021.

BEZERRA, I. C. et al. "Fui lá no posto e o doutor me mandou foi pra cá": processo de medicamentalização e (des)caminhos para o cuidado em saúde mental na atenção primária. Interface (Botucatu), São Paulo, v. 18, n. 48, p. 61-74. 2014. Disponível em: <http://www.scielo. br/pdf/icse/v18n48/1807-5762-icse-18-48-oo61. pdf $>$. Acesso: 22 mar. 2020.

BRAVO, M. I. S; PELAEZ, E. J; MENEZES, J. S. B. A saúde nos governos Temer e Bolsonaro: o SUS totalmente submetido ao mercado. In: Congresso Brasileiro de Assistentes Sociais, 16., 2019, Brasília, DF. Anais... Brasília, DF: Ginásio Nilson Nelson, 2019, p. 1-13. Disponível em: <https://broseguini.bonino.com.br/ojs/ index.php/CBAS/article/view/1878/1836>. Acesso em: 12 ago. 2020.

CECILIO, L. C. O.; REIS, A. A. C. Apontamentos sobre os desafios (ainda) atuais da atenção básica à saúde. Cadernos de Saúde Pública, Rio de Janeiro, v. 34, p. eooo56917, 2018. Disponível em: <https://www.scielo.br/pdf/csp/v34n8/1678-4464csp-34-o8-eooo56917.pdf>. Acesso em: 8 mar. 2021.

DALLA VECCHIA, M. D.; MARTINS, S. T. F. Desinstitucionalização dos cuidados a pessoas com transtornos mentais na atenção básica: aportes para a implementação de ações. Interface (Botucatu), São Paulo, v.13, n. 28, p. 151-164, 2009. Disponível em: <https://www.scielo.br/pdf/icse/ v13n28/v13n28a13.pdf>. Acesso em: 8 mar. 2021.

DAUMAS, R. P. et al. O papel da atenção primária na rede de atenção à saúde no Brasil: limites e possibilidades no enfrentamento da covid-19. Cadernos de Saúde Pública, Rio de Janeiro, v. 36, n. 6, p. eoo104120, 2020. Disponível em: <https://www.scielosp.org/article/csp/2020. v36n6/eoo104120/en/>. Acesso em: 27 ago. 2020.

DUARTE, M. Q. et al. Covid-19 e os impactos na saúde mental: uma amostra do Rio Grande do Sul, Brasil. Ciência \& Saúde Coletiva, Rio de Janeiro, v. 25, n. 9, p. 3401-3411, 2020. Disponível em: <https://www.scielo.br/pdf/csc/v25n9/1413-8123csc-25-09-3401.pdf >. Acesso em: 8 mar. 2021.

FIOCRUZ - FUNDAÇÃO OSWALDO CRUZ. Saúde mental e atenção psicossocial na pandemia covid-19: recomendações para gestores. Fiocruz, Brasília, DF, 2020. Disponível em: <https:// www.fiocruzbrasilia.fiocruz.br/wp-content/ uploads/2020/o4/Sa\%C3\%BAde-Mental-eAten $\% \mathrm{C}_{3} \% \mathrm{~A}_{7} \% \mathrm{C}_{3} \% \mathrm{~A}_{3}$ o-Psicossocial-na-PandemiaCovid-19-recomenda\% $3 \% \mathrm{C}_{7} \% \mathrm{C}_{3} \% \mathrm{~B}_{5}$ es-paragestores.pdf >. Acesso em: 10 mar. 2021.

FOUCAULT, M. O sujeito e o poder. In: DREYFUS, H.; RABINOW, P.; FOUCAULT, M. (Org.). Uma trajetória filosófica: para além do estruturalismo. Rio de Janeiro: Forense Universitária, 1995. p. 231-249.

GRYSCHEK, G.; PINTO, A. A. M. Saúde mental: como as equipes de Saúde da Família podem integrar esse cuidado na atenção básica? Ciência \& Saúde Coletiva, Rio de Janeiro, v. 20, n. 10, p. 3255-3263, 2015. Disponível em: <https://www.scielo.br/pdf/csc/v2on1o/1413-8123csc-20-10-3255.pdf>. Acesso em: 8 mar. 2021.

LANCETTI, A.; AMARANTE, P. D. C. Saúde mental e saúde coletiva. In: CAMPOS, G. W. S. et al. (Org.). Tratado de saúde coletiva. São Paulo: Hucitec, 2008. p. $615-634$.

LIMA, E. J. B. O cuidado em saúde mental e a noção de sujeito: pluralidade e movimento. In: SPINK, M. J. P.; FIGUEIREDO, P.; BRASILINO, J. (Org.). Psicologia social e pessoalidade. Rio de Janeiro: Centro Edelstein de Pesquisas Sociais, 2011. p. 109-134. Disponível em: <http://books.scielo.org/ $\mathrm{id} / \mathrm{xg}$ wwp/pdf/spink-9788579820571-o9.pdf>. Acesso em: 10 mar. 2021.

MATTOS, M. P. et al. Educação permanente em saúde nos Centros de Atenção Psicossocial: revisão integrativa de literatura. Saúde em Debate, Rio de Janeiro, v. 44, n. 127, p. 1271-1293, 2O20. DOI: 10.1590/0103-1104202012724

MERHY, E. E. et al. Rede básica, campo de forças e micropolítica: implicações para gestão e cuidado em saúde. Saúde em Debate, Rio de Janeiro, v. 43, n. especial, p. 70-83, 2019. Disponível em: 
<https://www.scielo.br/pdf/sdeb/v43nspe6/o103-1104sdeb-43-speo6-oo7o.pdf >. Acesso em: 8 mar. 2021.

MS - MINISTÉRIO DA SAÚDE. Departamento de Ações Programáticas Estratégicas. Departamento de Atenção Básica. Coordenação Geral de Saúde Mental. Coordenação de Gestão da Atenção Básica. Saúde mental e atenção básica: o vínculo e o diálogo necessários. Brasília, DF, 2003.

MS - MINISTÉRIO DA SAÚDE. Secretaria de Atenção à Saúde. DAPE. Coordenação Geral de Saúde Mental. Reforma psiquiátrica e política de saúde mental no Brasil. Documento apresentado à Conferência Regional de Reforma dos Serviços de Saúde Mental: 15 anos depois de Caracas. Brasília, DF: OPAS, 2005.

MOREL, A. P. M. Negacionismo da covid-19 e educação popular em saúde: para além da necropolítica. Trabalho, Educação e Saúde, Rio de Janeiro, v. 19, p. eoo315147, 2021. Disponível em: <http://www.revista.epsjv. fiocruz.br/upload/revistas/r1ooo.pdf >. Acesso em: 08 mar. 2021.

NABUCO, G.; OLIVEIRA, M. H. P. P.; AFONSO, M. P. D. O impacto da pandemia pela covid-19 na saúde mental: qual é o papel da atenção primária à saúde? Revista Brasileira de Medicina de Família e Comunidade, Rio de Janeiro, v. 15, n. 42, p. 2532, 2020. Disponível em: <https://rbmfc.org.br/rbmfc/ article/view/2532>. Acesso em: 10 mar. 2021.

ORNELL, F. et al. The impact of the COVID-19 pandemic on the mental health of healthcare professionals. Cadernos de Saúde Pública, Rio de Janeiro, v. 36. 2020. Disponível em:

<http://cadernos.ensp.fiocruz.br/csp/public_site/ arquivo/1678-4464-csp-36-o4-eooo63520.pdf>. Acesso: 23 abr. 2020.

SAMPAIO, M. L.; BISPO JÚNIOR, J. P. Dimensão epistêmica da Reforma Psiquiátrica Brasileira: significados de gestores, profissionais e usuários. Interface (Botucatu), São Paulo, v. 25, p. e200267, 2021. Disponível em: <https://www.scielo.br/pdf/ icse/v25/1807-5762-icse-25-e200267.pdf>. Acesso em: 8 mar. 2021.

SAMPAIO, M. L.; BISPO JÚNIOR, J. P. Entre o enclausuramento e a desinstitucionalização: a trajetória da saúde mental no Brasil. Trabalho, Educação e Saúde, Rio de Janeiro, v. 19, p. eoo313145, 2020. <https://www.scielo.br/pdf/tes/v19/1981-7746tes-19-eoo313145.pdf $>$. Acesso em: 8 mar. 2021.

SARAMAGO, J. Ensaio sobre a cegueira. São Paulo: Companhia das Letras, 1995.

SCHÜNKE, L. K.; GIONGO, C. R. Atravessamentos políticos: a cultura organizacional e o sofrimento moral no serviço público. Revista Psicologia Organizações e Trabalho, Brasília, v. 18, n. 3 , p. 449-456, 2018. Disponível em: <http://pepsic. bvsalud.org/pdf/rpot/v18n3/v18n3ao7.pdf>. Acesso em: 11 ago. 2020.

SILVA, B. N. et al. Reflexos das relações de saberpoder no contexto da estratégia de Saúde da Família. Archives of Health Investigation, [S.l.], v. 8, n. 5, p. 229-236, 2019. Disponível em: <https://pdfs. semanticscholar.org/bea2/611a76cbcog6fc25af45o egae374e773a2a9.pdf>. Acesso em: 8 mar. 2021.

SODRÉ, F. Epidemia de covid-19: questões críticas para a gestão da saúde pública no Brasil. Trabalho, Educação e Saúde, Rio de Janeiro, v. 18, n. 3, e00302134, 2020. Disponível em: <https://www.scielo.br/j/tes/a/YtCRHxTywqWm4 SChBHvqPBB/?lang=pt>. Acesso em: o8 mar. 2021.

WHO - WORLD HEALTH ORGANIZATION. Integrating Mental Health in Primary Care: A Global Perspective. Geneva, 2008. Disponível em: < https://www.who.int/ mental_health/resources/mentalhealth_PHC_2008.pd . Acesso em: 8 mar. 2021.

WHO - WORLD HEALTH ORGANIZATION. Mental Health and Psychosocial Considerations During the Covid-19 Outbreak. WHO.int, 18 mar. 2020. Disponível em: <https://www. who.int/publications/i/item/WHO-2O19-nCoVMentalHealth-2020.1>. Acesso em: 10 mar. 2021.

\section{Contribuições dos autores}

Mattos foi o responsável pela concepção, redação e revisão crítica do manuscrito. Pereira e Gomes trabalharam na redação e revisão crítica do manuscrito.

Recebido: 20/07/2021

Reapresentado: 13/03/2021; 20/07/2021

Aprovado: 27/09/2021 